Research paper

\title{
Effect of in situ modification of bacterial cellulose with carboxymethylcellulose on its nano/microstructure and methotrexate release properties
}

\author{
Marina de Lima Fontes ${ }^{\mathrm{a}}$, Andréia Bagliotti Meneguin ${ }^{\mathrm{a}, \mathrm{c}}$, Agnieszka Tercjak ${ }^{\mathrm{d}}$, Junkal Gutierrez ${ }^{\mathrm{d}}$, \\ Beatriz Stringhetti Ferreira Cury ${ }^{\mathrm{e}}$, Aline Martins dos Santos ${ }^{\mathrm{e}}$, Sidney J.L. Ribeiro ${ }^{\mathrm{b}}$, \\ Hernane S. Barud ${ }^{\mathrm{a}, \mathrm{b}, *}$ \\ ${ }^{a}$ University of Araraquara - UNIARA, 14801-320, Araraquara, SP, Brazil \\ b Institute of Chemistry, São Paulo State University - UNESP, 14801-970, Araraquara, SP, Brazil \\ ${ }^{c}$ Interdisciplinary Laboratory of Advanced Materials, Centro de Ciências da Natureza- CNN, Federal University of Piaui - UFPI, 64049-550, Teresina, PI, Brazil \\ ' Group 'Materials + Technologies' (GMT), Department of Chemical and Environmental Engineering, Engineering College of Gipuzkoa, University of the Basque Country \\ (UPV/EHU), Donostia-San Sebastián, Spain \\ e Department of Drugs and Pharmaceuticals, School of Pharmaceutical Sciences, SãoPaulo State University - UNESP, 14800-903, Araraquara, Sao Paulo, Brazil
}

\section{A R T I C L E I N F O}

\section{Keywords:}

Bacterial cellulose

In situ modification

Carboxymethylcellulose

Methotrexate

Controlled release

\begin{abstract}
A B S T R A C T
Bacterial cellulose/carboxymethylcelullose (BC/CMC) biocomposites with different DS-CMC (DS from 0.7 to 1.2 ) were developed in order to evaluate their impact as a drug delivery system. Biocomposites were loaded with methotrexate (MTX) as an alternative for the topical treatment of psoriasis. Scanning electron microscopy and atomic force microscopy showed that the CMC coated the cellulose nanofibers, leading to the decrease of the elastic modulus as the DS of CMC increased. BC/CMC0.9 exhibited the lower liquid uptake (up to 11 times lower), suggesting that the more linear structure of the intermediate substitute CMC grade (0.9) was able to interact more strongly with $\mathrm{BC}$, resulting in a denser structure. All samples showed a typical burst release effect in the first $15 \mathrm{~min}$ of test, however the BC/CMC0.9 biocomposite promoted a slight lowering of MTX release rates, suggesting that the DS of CMC can be considered the key factor to modulate the BC properties.
\end{abstract}

\section{Introduction}

The search for new biomaterials from green alternatives having specific properties has been the focus of many researchers worldwide, since they can be successfully explored for health purposes. In last decades, biomaterials have been intensively used for tissue engineering in order to replace or regenerate damaged tissues and their functions (Ribeiro, Sencadas, Correia, \& Lanceros-Méndez, 2015; Schenck et al., 2014). Moreover, they are commonly used in the pharmaceutical field to design new controlled drug delivery systems (CDDS), which promote the temporal and/or spatial control of drug release rates (Goonoo, Jeetah, Luximon-Bhaw, \& Jhurry, 2015; Kanamala, Wilson, Yang, Palmer, \& Wu, 2016; Liu, Zhan, Wan, Wang, \& Wang, 2015).

Unlike conventional dosage forms that release the drug immediately after administration (immediate-release), the CDDS modifies the time and/or release rates, keeping the plasma drug concentration within the therapeutic window, i.e., above the minimal effective concentration and below the minimal toxic concentration. All these features of CDDS contribute greatly to minimize fluctuations in the plasma drug concentration, promoting a more effective pharmacological therapy with reduced side effects (Sinha\&Kumria, 2003; Souto-Maior, Reis, Pedreiro, \& Cavalcanti, 2008).

Among several biopolymers of interest, cellulose is the most abundant and inexhaustible natural polymer on earth, and has numerous applications in different areas of technology. It is synthesized by some species of plants, fungi, marine animals (tunicates), mosses and bacteria. In particular, BC is synthesized by different species of bacteria, especially gram-negative bacteria, including Gluconacetobacter (originally Acetobacter), Agrobacterium, Aerobacter, Achromobacter, Azotobacter, Rhizobium and Salmonella (Chawla, Bajaj, Shrikant, Survas, \& Singhal, 2008; Shoda \& Sugano, 2005), and presents chemical purity, high surface area and Young's modulus, thermal stability and porosity. In addition, its well-organized 3D network of fibers contributes to the development of temporary skin substitutes, wound dressings and antimicrobial artificial blood vessels (Andrade et al., 2013; Fu et al., 2012). In pharmaceutical field, this material has been used as

\footnotetext{
* Corresponding author at: Institute of Chemistry, São Paulo State University - UNESP, 14801-970, Araraquara, SP, Brazil.

E-mail addresses: hsbarud@yahoo.com.br, hernane.barud@gmail.com (H.S. Barud).
} 


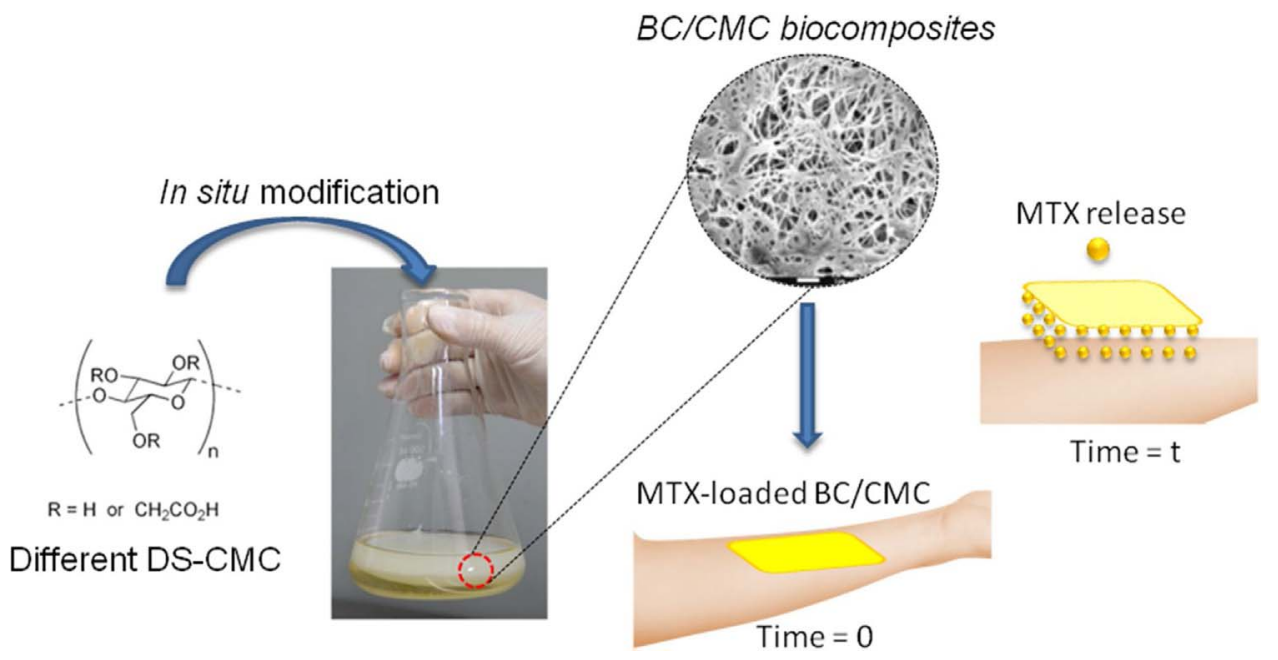

Scheme 1. Schematic illustration of the BC/CMC biocomposites preparation for topical release of MTX. emulsifier, dispersing agent, gelling agent (Donini et al., 2010), and successfully exploited in the design of CDDS (Almeida et al., 2014; Silva et al., 2014; Trovatti et al., 2012). The tailoring of BC properties, such as fibrils size, surface area and crystallinity, by in situ or ex situ modification of BC culture medium with different additives and concentrations has been evidenced, and now compiled into an important review article written by Stumpf, Yang, Zhang, and Cao (2016).

In this context, CMC can be introduced into BC culture medium and change its viscosity, interfering with the production rates and the overall properties of biocellulose, as early related by some researchers (Cheng, Jefrrey, \& Demirci, 2009; Ma, Zhao, Ji, Zeng, \& Li, 2014). Briefly, CMC is a water soluble cellulose derivative obtained by etherification of hydroxyl groups present in the cellulose molecule (Credou \& Berthelot, 2014). The physicochemical properties of CMC and its ability to interact with other substances depends on the DS, defined as the average number of carboxymethyl groups per monomeric unit, usually found in the range of 0.7 and 1.2 (Li et al., 2017).

Previous studies have demonstrated the effects of CMC addition on the BC structure formation. In situ modification of BC was carried out by Chen, Chen, Huang, and Lin (2011) in order to overcome the low BC rehydration capacity due to its high crystallinity. The main findings revealed that $\mathrm{CMC}$ led to the formation of a denser structure, but showing reduced mechanical strength and crystallinity. The authors believe that CMC adheres to the BC microfibrils in a discontinuous mode and interferes with the their aggregation, besides preventing the subsequent formation and crystallization of the $\mathrm{BC}$ ribbons. This behavior is responsible for the disorganized regions appearance which are able to improve the rehydration ratio of BC. Huang, Chen, Lin, Hsu, and Chen (2010) reported data similar to the previous ones, but an increase in bundle widths, followed by the decrease of network voids was verified. According to these authors, the mechanism by which CMC interferes with BC production is steric hindrance, which alters the formation of hydrogen bonds between microfibrils.

Another study focused on assessing the influence of CMC addition on BC production in a biofilm reactor for paper production (Cheng, Catchmark, \& Demirci, 2011). The surprising results revealed that the BC production was 1.7-fold enhanced when 1.5\% of CMC was applied as a result of the crystallinity and crystal size reduction, which are considered the limiting step in cellulose synthesis. Besides, it was noticed that in specific concentrations and conditions, CMC is able to change the produced BC from mat to pellets. Finally, Hirai, Tsuji, Yamamoto, and Horii (1998) examined the effect of different DS-CMC on the typical microfibrils ribbon assembly. When $0.1 \%$ CMC with DP 80 and DS 0.57 was applied, it was verified a greater effectiveness in the production of smaller microfibrils and narrower distribution of microfibril sizes. Furthermore, the raise of CMC concentration up to $1.0 \%$ led to increase of microfibrils size measuring $3-7 \mathrm{~nm}$ wide. The authors assume the increase of DS prevents the aggregation of the microfibrils into a normal ribbon assembly, probably by the adsorption of CMC onto the surface of the microfibrils through hydrogen bonding.

Despite the relevance of CMC in this area of research, few studies have addressed the influence of adding different DS-CMC in culture media for BC production (Hirai et al., 1998; Yamamoto et al., 1996), but no other assessed its impact on the $\mathrm{BC}$ performance as a drug delivery matrix. We hypothesize that CMC should interfere with the production of cellulose nanofibers in different rates according to its DS, either in the crosslinking density or overall network porosity, leading to the obtainment of nanostructured biocomposites with modulated properties, mainly the water uptake, mechanical and release properties. Therefore, in order to better understand the interactions between $\mathrm{BC}$ and $\mathrm{CMC}, \mathrm{BC} / \mathrm{CMC}$ biocomposites with different DS were prepared in this work by in situ modification of a static culture medium using Gluconacetobacter bacteria. MTX, a poorly water soluble-drug traditionally used in the treatment of cancer, inflammatory and autoimmune diseases (Wang et al., 2016) was incorporated to the BC/CMC membranes in order to optimize the topical treatment of psoriasis, an autoimmune disorder of skin characterized by hiperkeratosis and inflammation (Boakye et al., 2017), as shown in Scheme 1.

\section{Materials and methods}

\subsection{Materials}

Anhydrous D-glucopyranose and ethanol, citric acid, $\mathrm{KH}_{2} \mathrm{PO}_{4}$, $\mathrm{Na}_{2} \mathrm{HPO}_{4}, \mathrm{MgSO}_{4} \cdot 7 \mathrm{H}_{2} \mathrm{O}$, all P.A. grade were purchased from Synth . Bacteriologic peptone, agar and yeast extract were purchased from Merck. Sodium CMC with different degree of substitution (Theoretical $=0.7,0.9$ and 1.2; Experimental $=0.77,0.88$ and 1.09 and average degree of polymerization between 1400 and 1500, information determined from ASTM 1439-03) was purchased from Acros Organics. MTX was provided by Flagron Pharmaceutical (Sao Paulo, Brazil).

\subsection{Methods}

\subsubsection{Pre-inoculum}

Gluconacetobacter xylinus (ATCC 23769) was maintained in Hestrin and Schramm (HS) solid culture medium at $4-8{ }^{\circ} \mathrm{C}$. One single colony from the solid medium was used to inoculated into $50 \mathrm{~mL}$ of modified HS liquid medium, composed of $50 \mathrm{~g} / \mathrm{L}$ of glucose, $4 \mathrm{~g} / \mathrm{L}$ of yeast extract, $0.73 \mathrm{~g} / \mathrm{L}$ of $\mathrm{MgSO}_{4} \cdot 7 \mathrm{H}_{2} 0,2 \mathrm{~g} / \mathrm{L}$ of $\mathrm{KH}_{2} \mathrm{PO}_{4}, 20 \mathrm{~g} / \mathrm{L}$ of ethanol and distilled water $(1 \mathrm{~L})$. The set was incubated at $28{ }^{\circ} \mathrm{C}$ for $24 \mathrm{~h}$ in static 

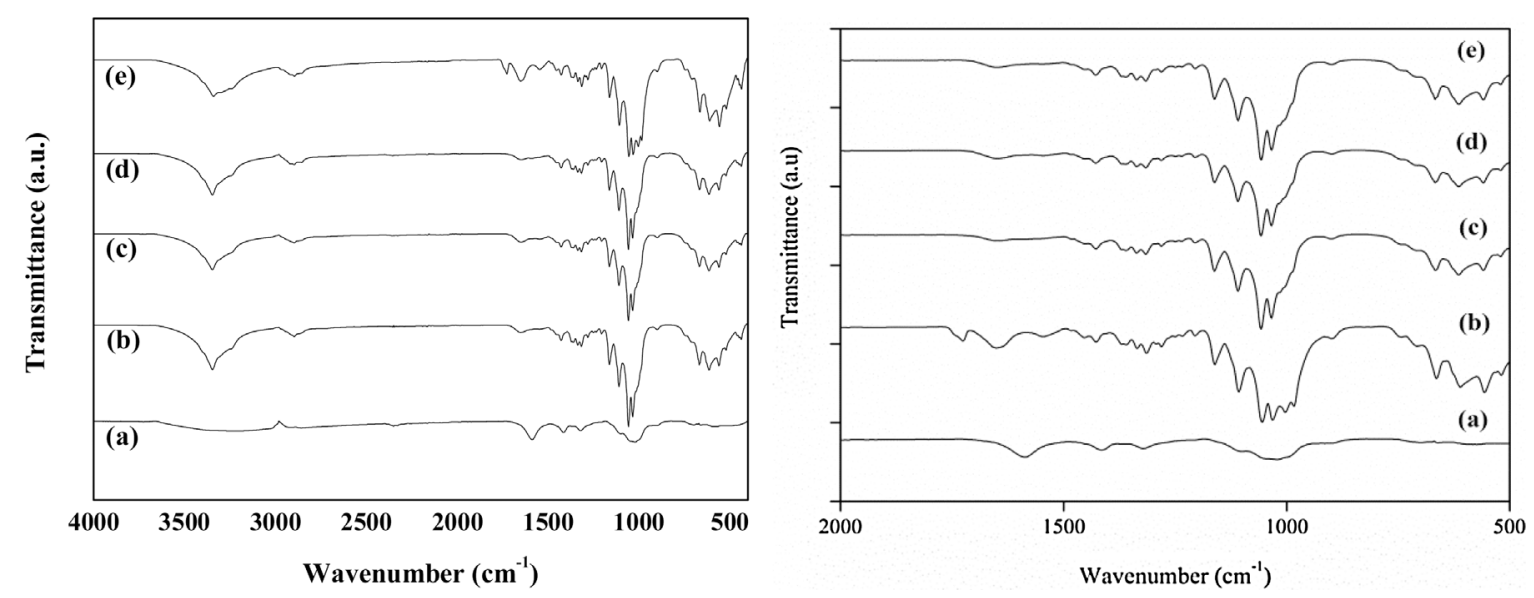

Fig. 1. FTIR spectra of (a) neat CMC DS 0.9, (b) neat BC membrane, (c) BC/CMC 0.7, (d) BC/CMC 0.9 and (e) BC/CMC 1.2 .

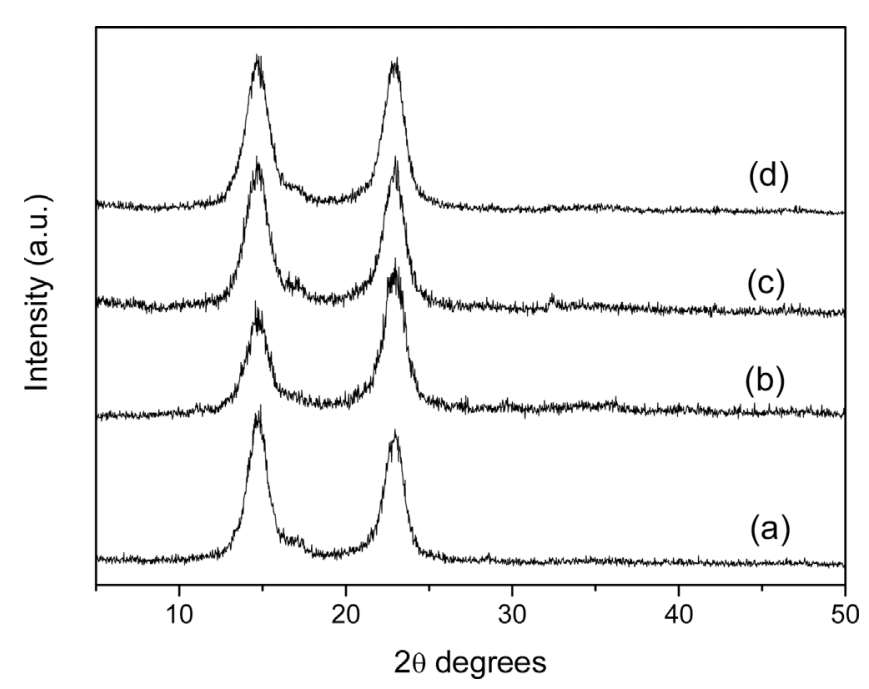

Fig. 2. X-ray diffraction of (a) neat BC membrane, (b) BC/CMC 0.7, (c) BC/CMC 0.9 and (d) BC/CMC 1.2.

condition.

\subsubsection{Biosynthesis of neat BC membrane and BC/CMC biocomposites}

$\mathrm{BC}$ membranes were produced by cultivating Gluconacetobacter $x y$ linus for three days at $28^{\circ} \mathrm{C}$, under static conditions, in $500 \mathrm{~mL}$ Erlenmeyer flasks containing $90 \mathrm{~mL}$ of HS medium and $10 \mathrm{~mL}$ of preinoculum. BC/CMC biocomposites were prepared analogous, but with the addition of $1 \%$ of CMC (DS $0.7,0.9$ or 1.2 ) in relation to the glucose mass. Subsequently, BC membranes and BC/CMC biocomposites were purified by treating them with $0.1 \mathrm{~mol} / \mathrm{L} \mathrm{NaOH}$ solution at $80^{\circ} \mathrm{C}$ for $30 \mathrm{~min}$. Finally, membranes were washed with purified water several times until neutral $\mathrm{pH}$.

Samples were labeled according to the DS of CMC added to the HS culture medium, as follows: BC/CMC0.7, BC/CMC0.9 and BC/CMC1.2.

\subsubsection{MTX-loading}

The samples were loaded with MTX by immersing BC membrane and $\mathrm{BC} / \mathrm{CMC}$ biocomposites in $4 \mathrm{~mL}$ MTX solution $(25 \mathrm{mg} / \mathrm{mL})$. Next, MTX-samples were dried in an oven with forced air circulation at room temperature until constant weight. For MTX loading determination, a known mass of samples was kept into ethanol/100 mM aqueous $\mathrm{NaOH}$ solution $(1 / 2, v / v)$ under mild magnetic stirring for $8 \mathrm{~h}$, followed by centrifugation at $5000 \mathrm{rpm}$ for $10 \mathrm{~min}$. MTX concentration in the supernatant was quantified in a spectrophotometer (Hewlett PackardKayak XA) by measuring the absorbance at $303 \mathrm{~nm}$ from a standard calibration curve (Wang \& Yan, 2017).

\subsubsection{Fourier transform infrared spectroscopy (FTIR)}

The freeze-dried samples were analyzed by FTIR performed in a Nicolet Nexus Spectra equipped with a Golden Gate single reflection diamond ATR accessory. The spectra were collected over the range of $4000-600 \mathrm{~cm}^{-1}$ with accumulation of 32 scans, resolution of $2 \mathrm{~cm}^{-1}$ and interval of $0.5 \mathrm{~cm}^{-1}$.

\subsection{5. $X$-ray diffraction (XRD)}

XRD patterns of samples were acquired on a Shimadzu XDR6000 diffractometer with $\mathrm{Cu}-\mathrm{K} \alpha$ radiation $(\lambda=1.5406 \AA), 2 \theta$ in the range between $10^{\circ}-30^{\circ}$ and a scan rate of $2^{\circ} \mathrm{min}^{-1}$. Crystallinity index (CrI) of the samples was calculated according to Segal method (Segal, Creely, Martin, \& Conrad, 1959) (Eq. (1)), as follows:

$C r I=\left(\frac{I_{002}-I_{a m}}{I_{002}}\right) \times 100$

where $I_{002}$ is the maximum intensity (in arbitrary units) of the 002 lattice diffraction and $\mathrm{I}_{\mathrm{am}}$ is the intensity of diffraction in the same units at $22.76^{\circ}(2 \theta)$.

\subsection{6. $T G / D T G$}

Thermogravimetric analyses of freeze-dried samples were performed using of TA SDT 2960 (TA Instruments, USA). All samples (about $7 \mathrm{mg}$ ) were heated in alumina pans from $25^{\circ} \mathrm{C}$ to $600{ }^{\circ} \mathrm{C}$ temperature range under nitrogen atmosphere (flow rate of $100 \mathrm{~mL} \mathrm{~min}^{-1}$ ), at a heating rate of $10{ }^{\circ} \mathrm{C} \mathrm{min}^{-1}$.

\subsubsection{Field emission gum scanning electron microscopy (FEG-SEM)}

The microstructure of air-dried samples $\left(30^{\circ} \mathrm{C}\right.$ for $48 \mathrm{~h}$ ) was characterized by FEG-SEM on a JEOL microscope (model JSM-7000F). The samples were attached to slab surfaces with double sided adhesive tape and then coated with a layer of carbon. All samples were examined using an accelerating voltage of $2 \mathrm{kV}$.

\subsubsection{PeakForce quantitative NanoMechanics atomic force microscopy (PeakForce QNM-AFM)}

PeakForce QNM-AFM was used to study nanomechanical properties of air-dried samples $\left(30^{\circ} \mathrm{C}\right.$ for $48 \mathrm{~h}$ ) using Dimension Icon microscope from Bruker. Measurements were carried out in PeakForce mode under ambient conditions. A silicon tip with nominal radius of $10 \mathrm{~nm}$, cantilever length of $125 \mu \mathrm{m}$, and resonance frequency of $150 \mathrm{kHz}$ was used. The measurements were performed with a calibrated optical sensitivity. The exact spring constant of the tip was calculated using the Thermal Tune option and a defined tip radius was adjusted using PS as standard. 

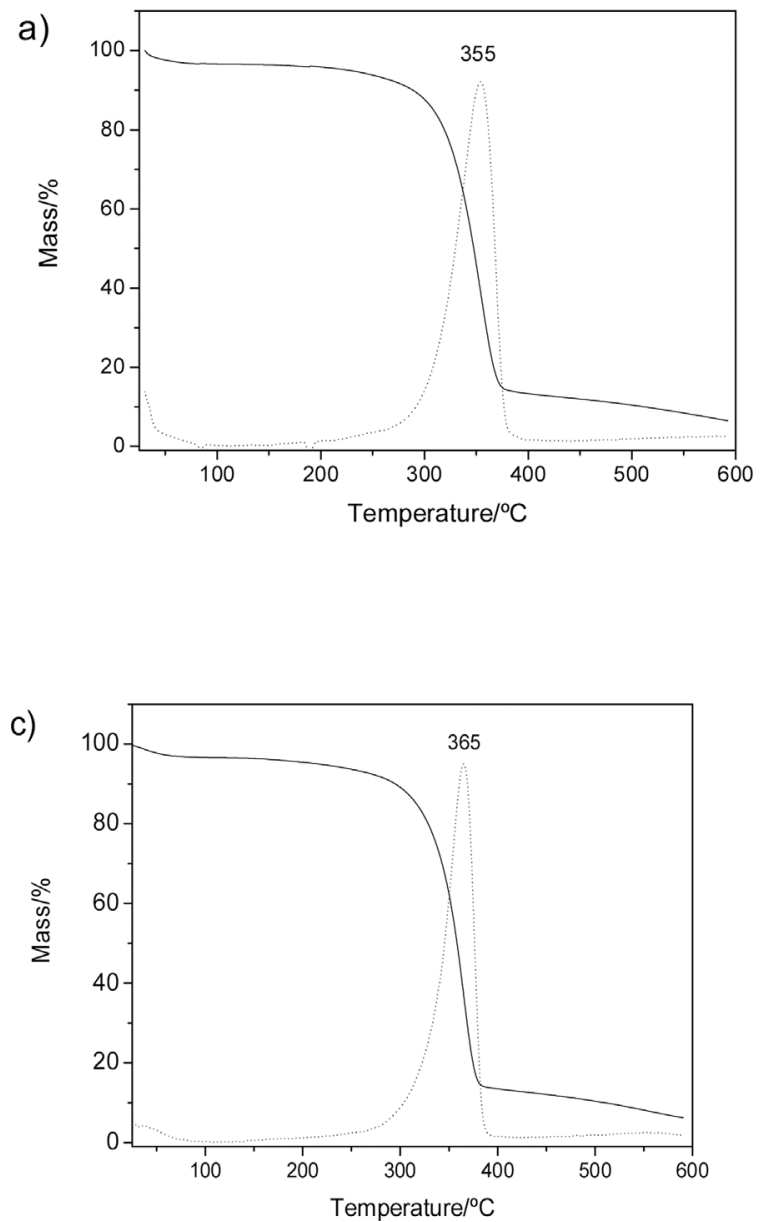

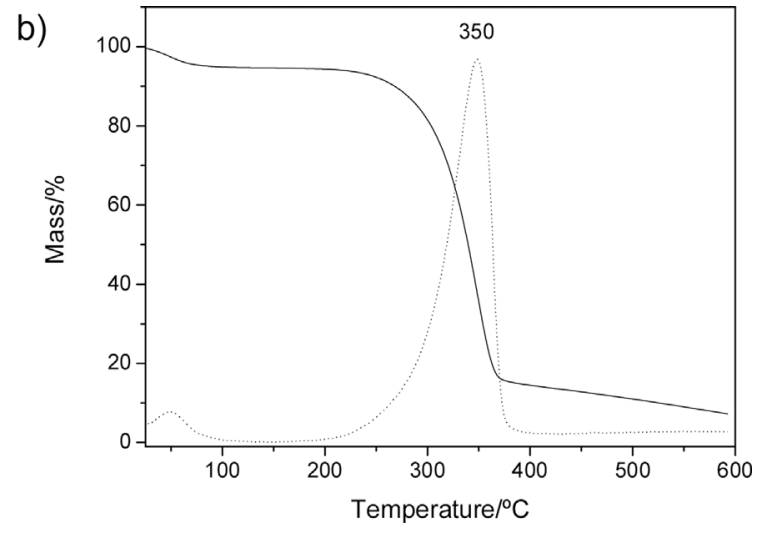

d)

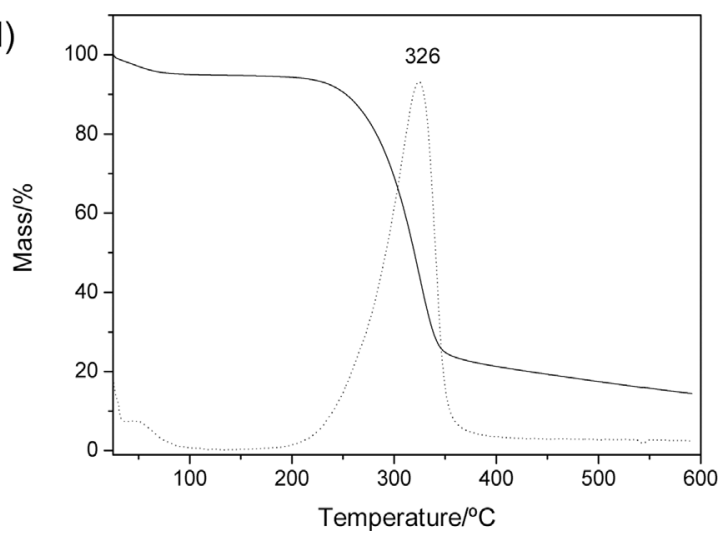

Fig. 3. TGA curves: (a) neat BC membrane, (b) BC/CMC 0.7, (c) BC/CMC 0.9 and (d) BC/CMC 1.2 .

\subsubsection{Liquid uptake test}

The profile of liquid uptake of samples was evaluated on an Enslin's device according to Meneguin, Cury, and Evangelista (2014) and Voigt (2000). Pieces of samples (around $1 \mathrm{~cm}^{2}$ ) were accurately weighed and carefully placed on the sintered glass filter of funnel. The changes on volume of $0.1 \mathrm{~mol} / \mathrm{L}$ phosphate buffered saline solution $(\mathrm{pH} \mathrm{7.4)}$ were evaluated at $2,7,17,27,42,57,82,120 \mathrm{~min}$. Results were expressed as medium absorbed (\%) in relation to initial mass of sample and the tests were carried out in triplicate. The liquid uptake data were fitted with Korsmeyer-Peppas, Higuchi and Weibull mathematical model with the aid of Sigma Plot 10.0 software.

\subsection{In vitro $M T X$ release}

Dissolution tests of MTX-loaded samples were performed on a Hanson Research dissolution station (New Hanson SR-8 Plus), equipped with a paddle over the disk apparatus (USP V) (USP 29, 2006), satisfying the sink conditions. The samples containing $100 \mathrm{mg}$ of MTX were carefully fixed on the transdermal patch holder and placed in the bottom of vessels containing $900 \mathrm{~mL}$ of $0.1 \mathrm{~mol} / \mathrm{L}$ phosphate buffer $(\mathrm{pH}$ 7.4), stirred at $50 \mathrm{rpm}$, at $37 \pm 0.4^{\circ} \mathrm{C}$, for $180 \mathrm{~min}$. Aliquots of $2 \mathrm{~mL}$ were withdrawn in predetermined times and replaced by fresh medium at the same temperature. Samples were filtered through $0.45 \mu \mathrm{m}$ nylon filter prior to analysis. The MTX release was quantified by measuring the absorbance at $303 \mathrm{~nm}$. Dissolution studies were performed in triplicate.

\section{Results and discussion}

\subsection{FTIR}

FTIR spectrum of CMC, biosynthesized neat BC membrane and BC/ CMC biocomposites are shown in Fig. 1. The FTIR spectrum of CMC (Fig. 1a) shows bands at $3300 \mathrm{~cm}^{-1}$ attributed to the $\mathrm{O}-\mathrm{H}$ stretching region, at $\sim 1587 \mathrm{~cm}^{-1}$ assigned to stretching of the carboxyl group, $-\mathrm{CH}_{2}$ scissoring at $\left(1414 \mathrm{~cm}^{-1}\right)$ and $\mathrm{OH}$ stretching in-plane at $1318 \mathrm{~cm}^{-1}$ (Chai \& Isa, 2013). The band at $1020 \mathrm{~cm}^{-1}$ corresponds to carboxymethyl ether group stretching (Hashem, Sharaf, ElHady, \& Hebeish, 2013; Juncu, Stoica-Guzun, Stroescu, Isopencu, \& Jinga, 2016; Li et al., 2009).

The typical bands observed for the BC membrane (Fig. 1b) can be attributed to $\mathrm{O}-\mathrm{H}$ stretching vibration of cellulose type $\mathrm{I}\left(3340 \mathrm{~cm}^{-1}\right)$ (Moharram \& Mahmoud, 2008), C-H stretching of the $\mathrm{CH}_{2}$ and $\mathrm{CH}_{3}$ groups ( $2898 \mathrm{~cm}^{-1}$ ) (Oh et al., 2005) and $\mathrm{H}-\mathrm{O}-\mathrm{H}$ bending of adsorbed water $\left(1650 \mathrm{~cm}^{-1}\right.$ ) (Barud et al., 2008). According to Meneguin et al. (2017), other bands in the range of $\left(1200-900 \mathrm{~cm}^{-1}\right)$ are related to $\mathrm{C}-\mathrm{O}$ and $\mathrm{C}-\mathrm{C}$ vibration bonds of glycosidic bonds and pyranoid rings.

In general, the same bands have been seen for neat $\mathrm{BC}$ membranes and $\mathrm{BC} / \mathrm{CMC}$ biocomposites, indicating that the cellulose derivative addition did not promote changes in the chemical structure of biocomposites, so that the biocomposites spectra represent the sum of the characteristic bands of each isolated polymer.

\section{2. $X R D$}

$\mathrm{XRD}$ patterns of neat $\mathrm{BC}$ membrane and all $\mathrm{BC} / \mathrm{CMC}$ biocomposites 

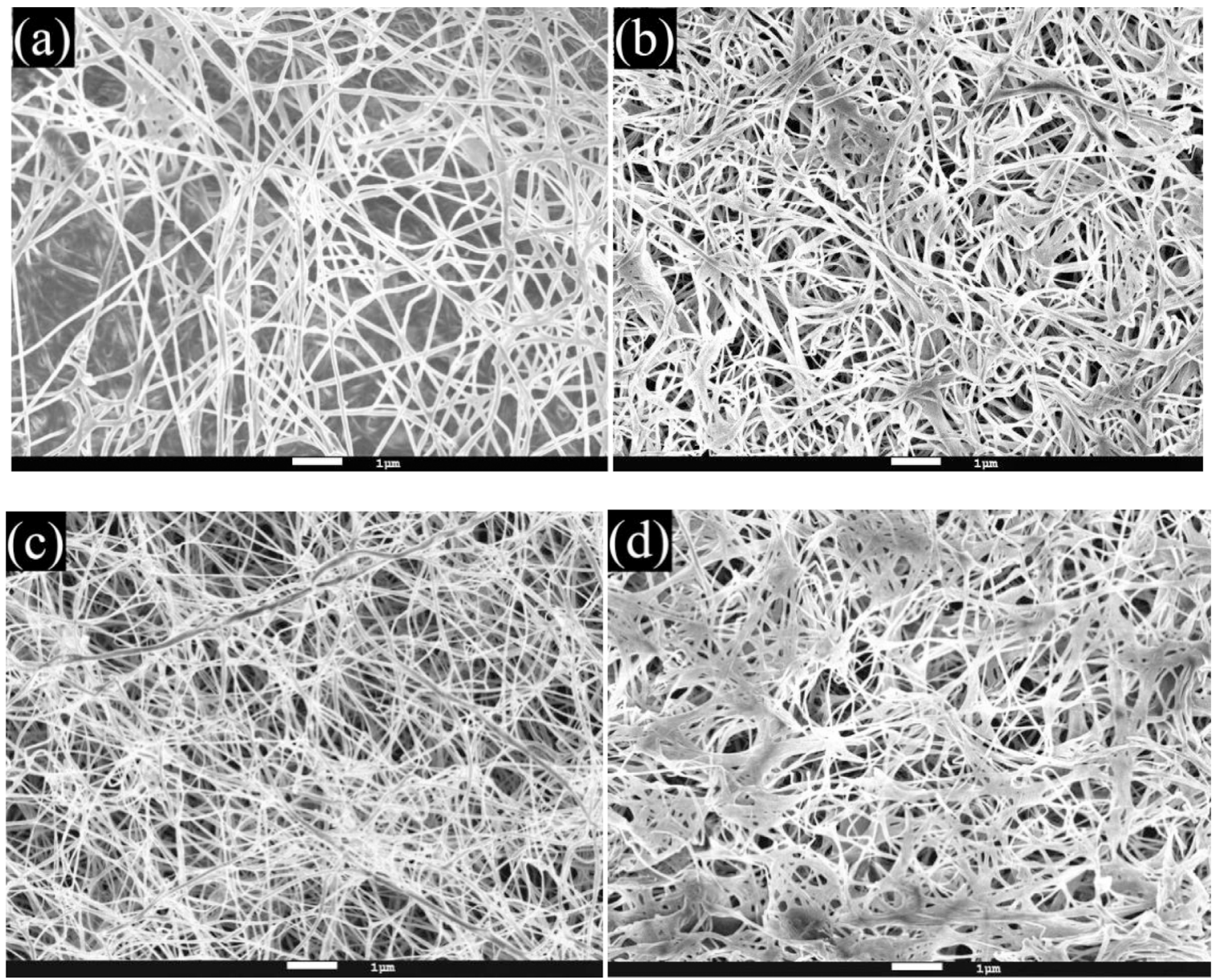

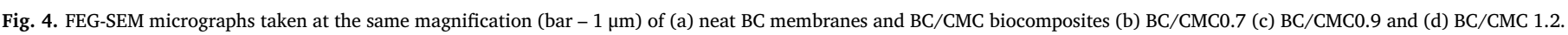

are depicted in Fig. 2. BC membrane (Fig. 2a) exhibited broad peaks at 14.8 and $22.5^{\circ}(2 \theta)$ related to the $(1 \overline{1} 0)$ and $(200)$ planes, respectively (Lima et al., 2015), which are assigned to the interplanar spacing of type 1 native cellulose (Kim, Kim, Wee, Park, \& Ryu, 2006).

The BC/CMC biocomposites diffractograms showed no significant changes in relation to neat $\mathrm{BC}$ membrane, indicating that the in situ modification of the culture medium using soluble cellulose derivative did not alter the crystallinity pattern of BC matrix.

Concerning CrI, which expresses the relative degree of crystallinity (Segal et al., 1959), it was observed a slight decrease for biocomposites in relation to neat $\mathrm{BC}$ membrane $(89.2 \%$ against 88.5 , 88.5 and $88.1 \%$ for $\mathrm{BC} / \mathrm{CMC} 0.7, \mathrm{BC} / \mathrm{CMC} 0.9$ and $\mathrm{BC} / \mathrm{CMC1.2}$, respectively). This behavior can be attributed to the viscosity increase of the CMC-mediated culture medium, as well as to the interaction of CMC with the cellulosic nanofibers during the crystallization process, resulting in lower crystallinity of the polymer (Cheng et al., 2011).

\section{3. $T G / D T G$}

In Fig. 3, TG/DTG curves for neat BC membrane and BC/CMC bicomposites are demonstrated. All samples showed very similar behavior with two main events of mass loss. The first one (about 8\%) occurred in a temperature range between 25 and $200{ }^{\circ} \mathrm{C}$, related to water desorption due to heating process. The second and main degradation stage $(67-75 \%)$ begins at $250{ }^{\circ} \mathrm{C}$, with maximum decomposition $\left(t_{\text {peak }}\right)$ at $355,350,365$ and $326^{\circ} \mathrm{C}$ for neat BC membrane, BC/CMC 0.7, BC/CMC 0.9 and $\mathrm{BC} / \mathrm{CMC} 1.2$, respectively. This event is attributed to the depolymerization and decomposition of dehydrocellulose into gases (Barud et al., 2007).

\subsection{FEG-SEM}

Fig. 4 shows FEG-SEM micrographs of neat BC membrane and BC/ CMC biocomposites. The surface of all samples shows the characteristic three-dimensional network of cellulose interpenetrating nanofibers forming a highly porous system, which is responsible for the excellent mechanical properties seen in this biopolymer, as well as the high water absorption capacity and liquid retention.

Although the crosslinking density of the cellulosic network has become denser in the BC/CMC biocomposites, they did not exhibit a notable change in the nanofibers morphology in terms of length and thickness when compared to neat BC membranes. However, CMC was able to modify the surface of the biocomposites, since they presented on their surface specific points that possibly represent the CMC coating the nanofibers, as can be seen mainly in Fig. 4b and d. Moreover, it can be observed that the CMC incorporation into $\mathrm{BC}$ nanofibers led to the reduction of the biocomposites porosity, as also observed by Ma et al. (2014), as a simple result of CMC free diffusion in the BC membrane.

\subsection{PeakForce (QNM-AFM)}

Atomic force microscopy in PeakForce mode was used to determine quantitative nanomechanical properties (QNM) of neat BC membranes and BC/CMC biocomposites at the nanoscale level (Fig. 5). AFM height images of the samples showed typical interconnected nanofibers network characterized for BC. Similarly as observed by FEG-SEM results discussed above, the average size of the nanofibers did not change considerably with increase of CMC DS in BC/CMC biocomposites. However, from comparison of the AFM height images, one can clearly observed presence of other material uniformly dispersed on the 

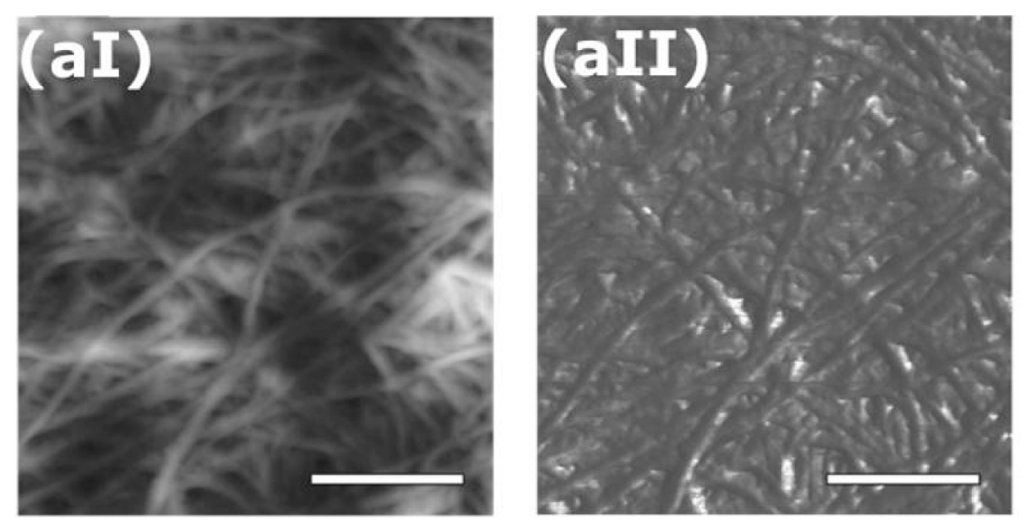

\section{(aIII)}
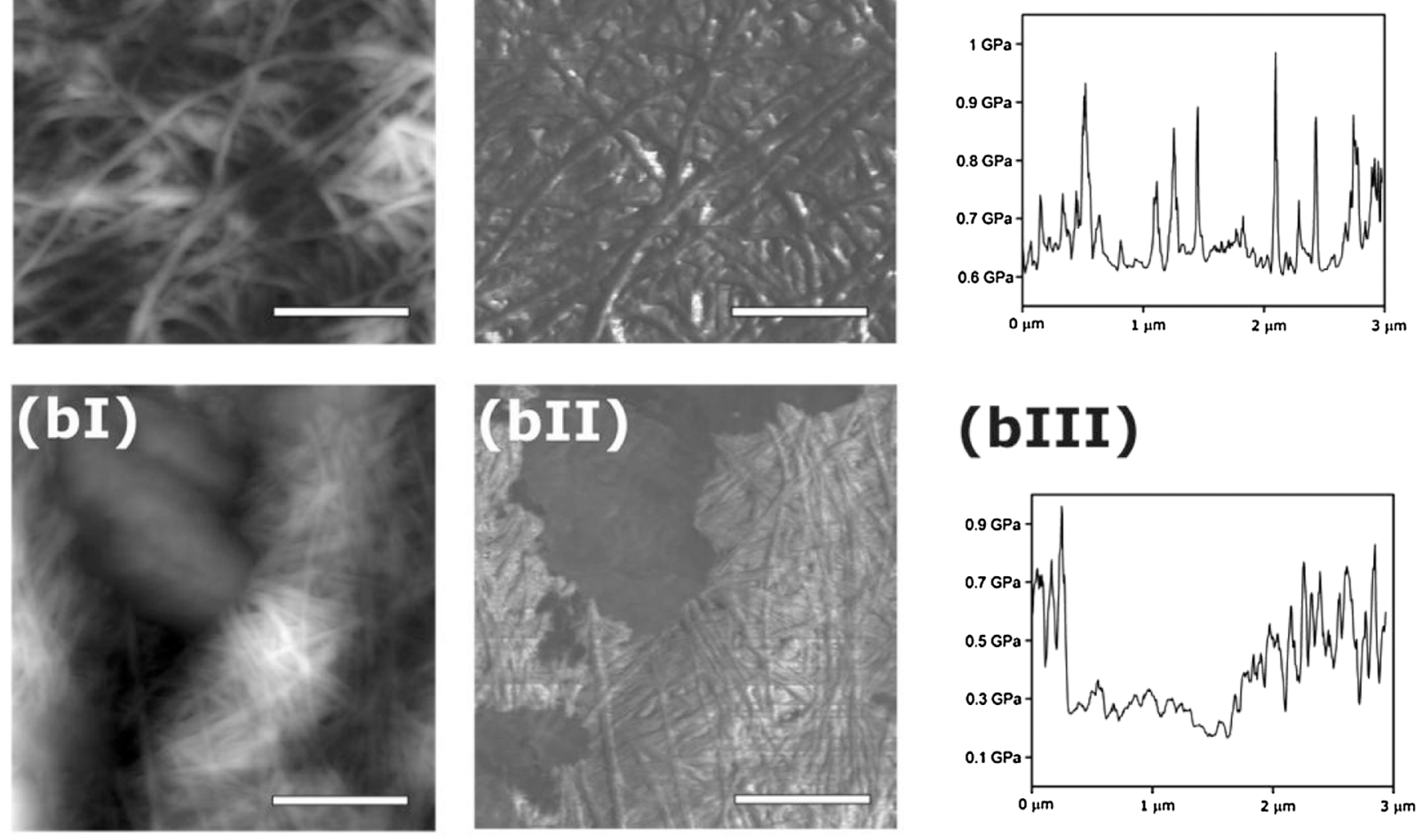

\section{(bIII)}
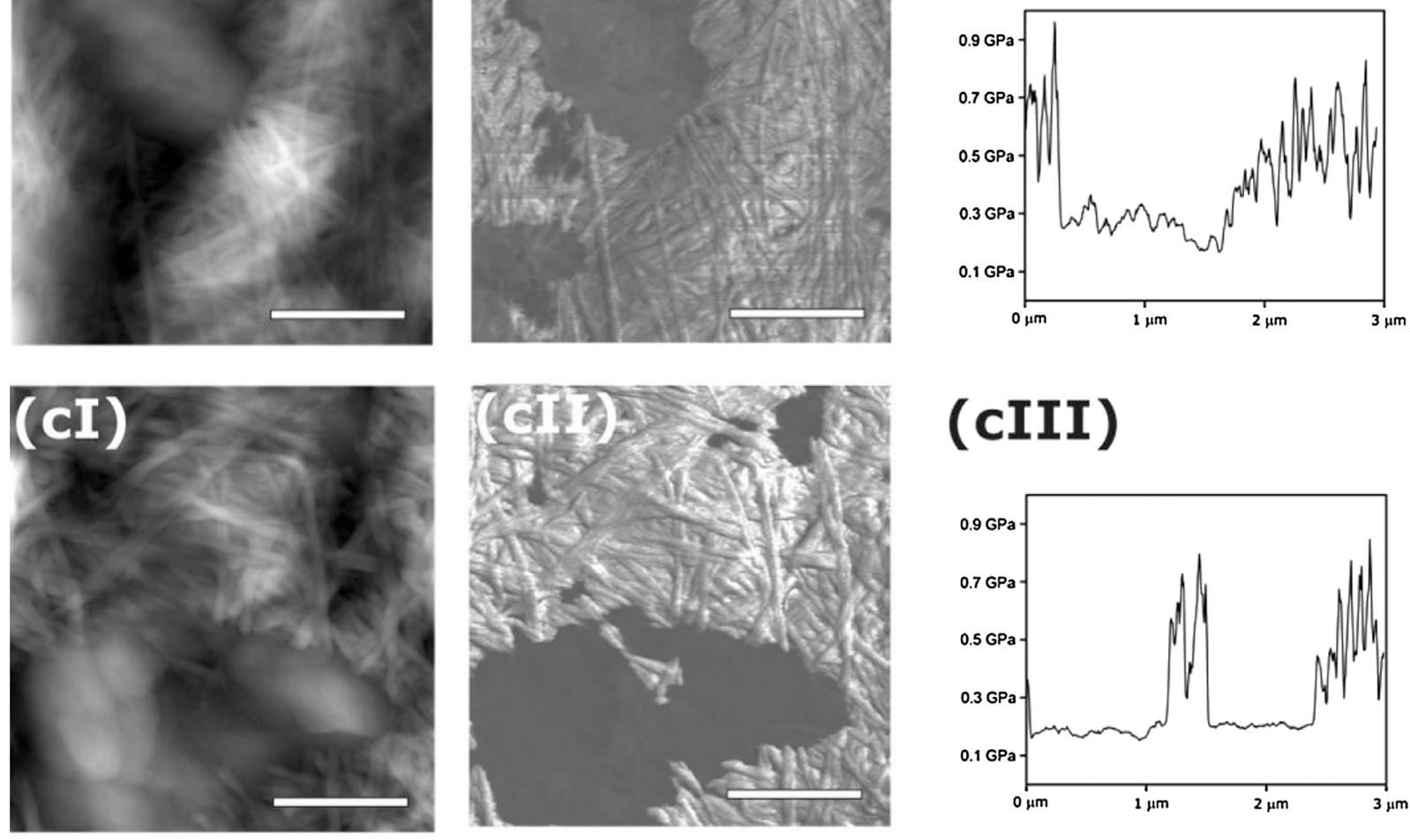

\section{(cIII)}
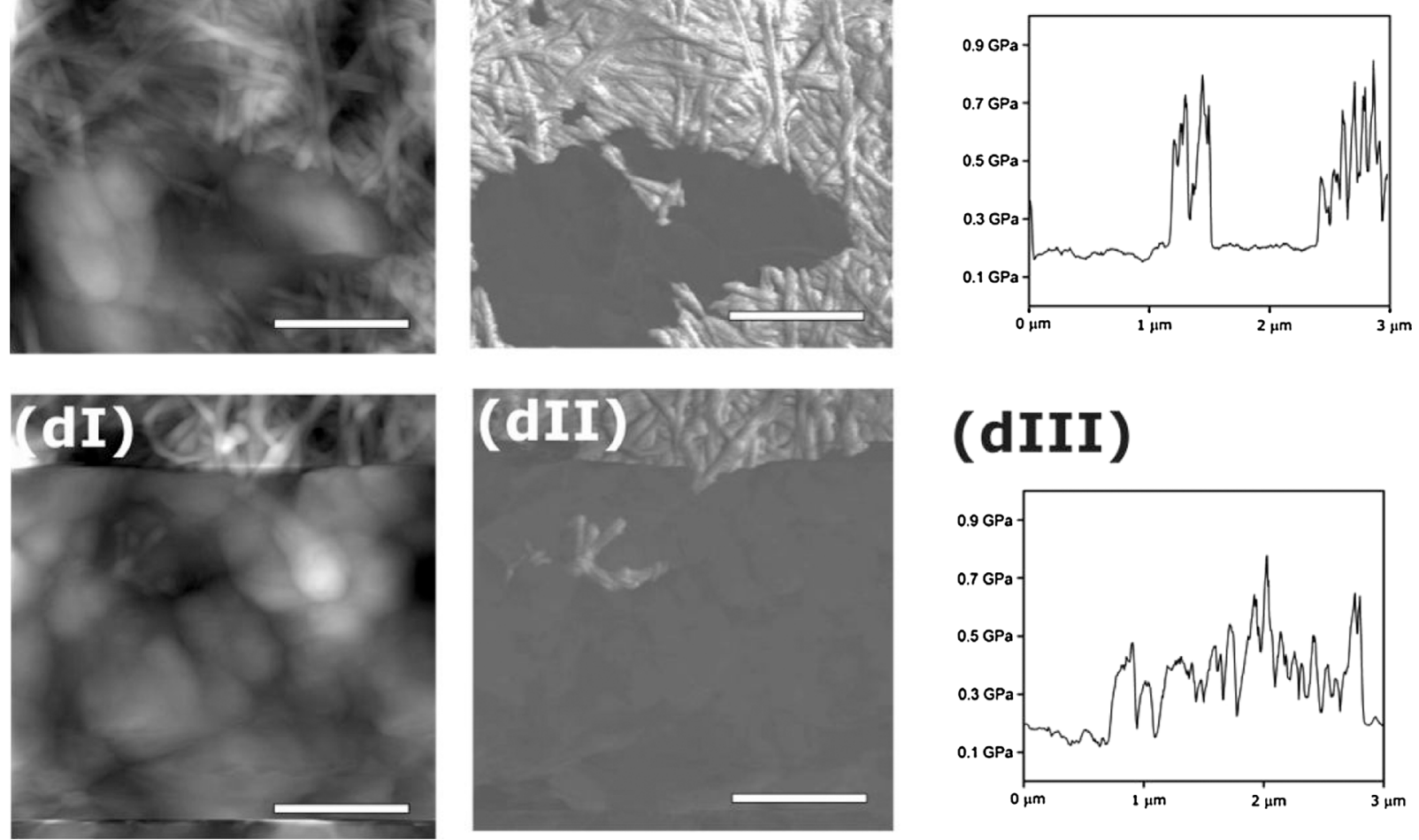

\section{(dIII)}

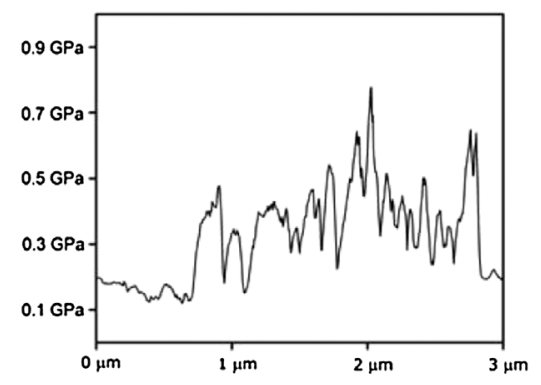

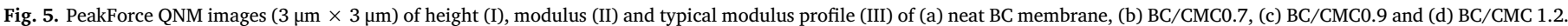
Typical profiles of each sample correspond to elastic modulus. 


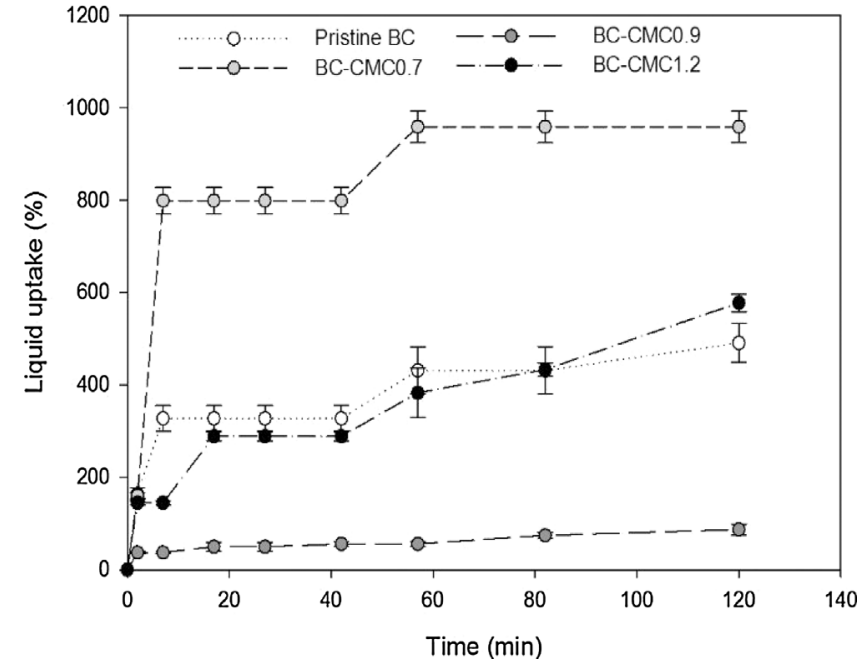

Fig. 6. Liquid uptake profile of neat BC membrane and BC/CMC biocomposites evaluated in $0.1 \mathrm{~mol} / \mathrm{L}$ phosphate buffer (pH 7.4) for $120 \mathrm{~min}$, expressed in percentage (\%).

Table 1

Values of correlation coefficients $\left(r^{2}\right)$ of liquid uptake data for different mathematical models (Higuchi, Korsmeyer-Peppas and Weibull) in phosphate buffer (pH 7.4).

\begin{tabular}{llcc}
\hline Samples & Korsmeyer-Peppas & Weibull & Higuchi \\
\hline Neat BC & $\mathbf{0 . 9 7 1 7}$ & 0.8487 & 0.7863 \\
BC-CMC0.7 & $\mathbf{0 . 9 6 0 9}$ & 0.9225 & 0.7158 \\
BC-CMC0.9 & $\mathbf{0 . 9 6 9 2}$ & 0.7892 & 0.8660 \\
BC-CMC1.2 & $\mathbf{0 . 9 7 6 8}$ & 0.8854 & 0.9584 \\
\hline
\end{tabular}

Table 2

$K$ and $n$ values obtained from liquid uptake data in phosphate buffer (pH 7.4) for $120 \mathrm{~min}$.

\begin{tabular}{lll}
\hline Sample & $K\left(\% \mathrm{~min}^{-\mathrm{n}}\right)$ & $n$ \\
\hline Neat BC & 173.54 & 0.2106 \\
BC-CMC0.7 & 387.21 & 0.2084 \\
BC-CMC0.9 & 23.67 & 0.2503 \\
BC-CMC1.2 & 76.06 & 0.4067 \\
\hline
\end{tabular}

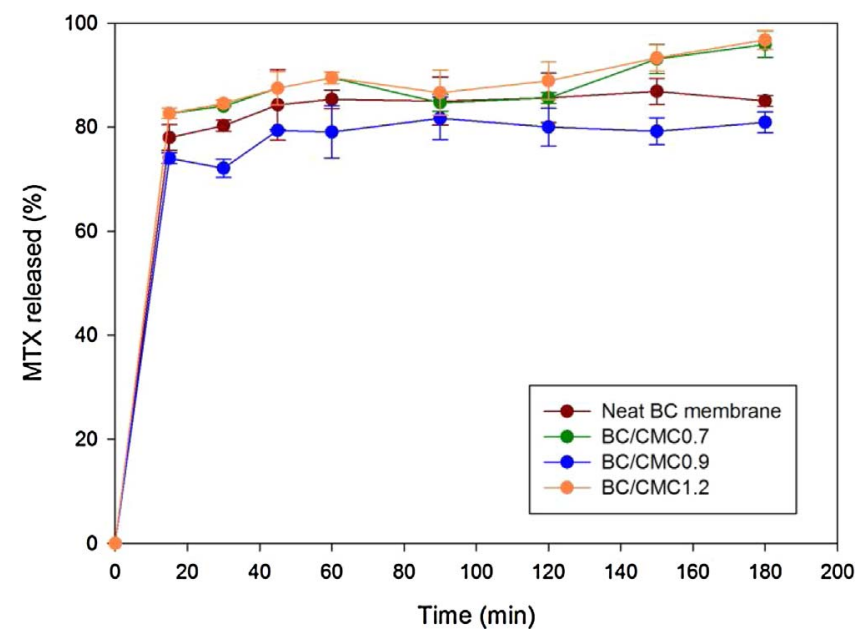

Fig. 7. In vitro MTX release profiles neat $\mathrm{BC}$ membrane and $\mathrm{BC} / \mathrm{CMC}$ biocomposites in phosphate buffer ( $\mathrm{pH} 7.4)$.

analyzed surface of the $\mathrm{BC} / \mathrm{CMC}$ biocomposites, which covered the nanofibers. Taken into account that the surface covered by this material increased with increase the DS of CMC one can concluded that this material correspond to CMC homogeneously spread on the BC membranes. Independently on the quantity of the CMC phase, regular globular morphology is detected for these areas on the surface of the investigated membranes. The average size of this structure is $80 \pm 10 \mathrm{~nm}$ in diameter.

The AFM elastic modulus images of neat $\mathrm{BC}$ membrane indicated the elastic modulus around $0.9 \mathrm{GPa}$. In this case, similar elastic modulus is uniformly distributed on the whole analyzed surface. Addition of CMC into BC/CMC biocomposites provoked significant changes in local nanomechanical properties. The average modulus corresponds to $3 \mu \mathrm{m} \times 3 \mu \mathrm{m}$ scanned area decrease with increasing of DS of CMC in $\mathrm{BC} / \mathrm{CMC}$ biocomposites being average elastic modulus equal to $800 \mathrm{MPa}, 700 \mathrm{MPa}$ and $500 \mathrm{MPa}$ for BC/CMC 0.7, BC/CMC 0.9 and BC/ CMC 1.2, respectively.

The decrease of the average elastic modulus is strongly related to low elastic modulus of the CMC phase, which can be clearly seen comparing the profiles corresponding to elastic modulus of each analyzed membranes. The BC nanofibers covered by $\mathrm{CMC}$ showed local elastic modulus equal to $300 \mathrm{MPa}$ for BC/CMC 0.7 and $200 \mathrm{MPa}$ for $\mathrm{BC} /$ CMC 0.9 and BC/CMC 1.2. Moreover, the local elastic modulus corresponds to $\mathrm{BC}$ phase in $\mathrm{BC} / \mathrm{CMC}$ bionanocomposites decrease slightly with increase DS of CMC (compare profiles corresponding to the modulus of each investigated membrane) being $\sim 0.7 \mathrm{GPa}$ for $\mathrm{BC} / \mathrm{CMC}$ 0.7 and $\mathrm{BC} / \mathrm{CMC} 0.9$ and $0.6 \mathrm{GPa}$ for BC/CMC 1.2. Mechanical properties at the nanoscale depended strongly on the DS of CMC in analyzed biocomposites. As the elastic modulus always decreases with the increase of DS-CMC, both factors should contribute to this behavior, namely the inherent low modulus of the CMC, as well as the increase of the substituent groups in the glycopyranose molecule that may be responsible for decreasing the inter/intra-chains interactions of the cellulose by hydrogen bonds.

\subsection{Liquid uptake test}

The study of liquid uptake capacity is a simple, but valuable characterization tool that provides important insights about the basic properties of polymeric systems, which definitively influence their final properties, such as mechanical and drug release properties. In addition to the high liquid uptake rates (e.g., water) are a strong indicative of the preferably hydrophilic character of the system, they can make inferences on the entanglement degree of structures and the interactions occurring between polymeric chains and solvent (Bouklas \& Huang, 2012). Thus, these results have a deep importance on the release kinetics of CDDS, which may have the release triggered by swelling, occurring in isolation or in conjunction with other mechanisms, such as diffusion and erosion (Siepmann \& Peppas, 2011). Swelling is a mechanism of release control induced by the diffusion of water molecules or biological fluid into dosage form, making the polymer chains more relaxed and flexible, followed by a volume expansion, facilitating the drug diffusion of the device (Brannon-Peppas, 1990a, 1990b). Thus, drug release rates from swellable systems are frequently associated to the speed of swelling process, as well as to the thickness and viscosity degree of the gel layer built around the swollen dosage form.

Neat $\mathrm{BC}$ membranes and BC/CMC biocomposites showed different liquid uptake profiles (Fig. 6), so that the BC/CMC0.7 sample showed the highest liquid uptake (about 958\%), while BC/CMC 0.9, BC/CMC 1.2 and neat BC samples exhibited values of $86 \%, 578 \%$ and $490 \%$, respectively. These results show that the DS of CMC affect the $\mathrm{BC}$ microstructure, so that molecular structure of the low substituted CMC grade (0.7) arranged in a coiled chain (Caraschi \& Campana, 1999) contributes to increase of medium viscosity and thus building a more porous fiber network, which facilitates the liquids absorption. In relation to the reduced liquid uptake demonstrated by BC/CMC 0.9, the more linear structure of intermediate CMC grade (0.9) should have allowed a stronger interaction among their ester and the hydroxyls groups of $\mathrm{BC}$, contributing to the formation of a denser and stiffer network that opposes to the liquid penetration. 
Liquid absorption kinetics of samples was evaluated from the data obtained in $0.1 \mathrm{~mol} / \mathrm{L}$ phosphate buffer $(\mathrm{pH} 7.4)$ for $120 \mathrm{~min}$. Korsmeyer-Peppas, Weibull and Higuchi mathematical models were applied to the results of liquid uptake in order to assess the dynamics of liquid penetration in the neat $\mathrm{BC}$ membranes and $\mathrm{BC} / \mathrm{CMC}$ biocomposites. The adjusted correlation coefficients $\left(r^{2}\right)$ are shown in Table 1. Since the $r^{2}$ is a quality parameter of applied model in relation to its ability to estimate correctly the responses, $r^{2}$ values near to 1indicate an higher correlation with the analyzed data.

According to $r^{2}$ values exhibited in Table 1, all samples fitted better with Korsmeyer-Peppas, which is based on the Power Law. The kinetic constant of liquid penetration $(k)$ and the exponent $n$, which is indicative of the liquid penetration mechanism according to geometrical shape of device, were calculated by the Korsmeyer-Peppas equation $\left(M_{t} / M_{\infty}=k t^{n}\right)$, where $M_{t}$ and $M_{\infty}$ are the absorbed medium volume at time $t$ and in the steady state, respectively.

All samples presented $n$ values in the range of $0.2084-0.4067$ (Table 2), which indicates that liquid uptake was driven by Fickian diffusion (case-I) for thin films, i.e, the liquid penetration is governed by water molecules diffusion into polymeric matrix according to concentration gradient (Ritger \& Peppas, 1987). In a comparative analysis, the $k$ values range between 23.67 and $387.21 \% \mathrm{~min}^{-\mathrm{n}}$, which indicates that liquid uptake rate of BC-CMC 0.9 sample was about 7.3 times lower than neat BC, while for BC-CMC 0.7 it was about 2.2 times higher. Moreover, the high $k$ values indicate that all samples (except BC-CMC 0.9) showed a burst effect of swelling (Gil, Colarte, Bataille, Brouillet, \& Caraballo, 2009). The set of data suggests that degree of substitution of CMC should provide a strong influence on BC microstructure and thus on the swelling properties of $\mathrm{BC}$ membranes.

\subsection{In vitro $M T X$ release}

Dissolution profiles of MTX from neat BC membrane and BC/CMC biocomposites are shown in Fig. 7. A burst effect of release was observed during the first 15 min of the test, in which about $70-80 \%$ of the drug was released from all samples. After this, a steady release of MTX was reached. The burst effect observed was related to the rapid diffusion of MTX molecules dispersed between cellulosic nanofibers, as a result of the large surface area originated by three-dimensional porous structure of bacterial cellulose that contributes to the faster drug release (Meneguin et al., 2017; Wei et al., 2011). MTX release from BC/CMC0.7 after 180 min of test was higher (96\%) than from BC/CMC0.9 (80\%) ( $\mathrm{p}<0.05$ ), which can be related to its higher swelling capacity due to its coiled chain that facilitates the drug molecules diffusion by the relaxation of polymeric chains and formation of a looser structure. Likewise, the lowest drug release rate presented by more linear BC/CMC 0.9 showed that the intermediate substitute CMC (0.9) probably allowed a higher crosslinking of the nanofiber network and a greater control over drug release.

\section{Conclusions}

BC/CMC biocomposites were successfully obtained after the in situ modification of the standard HS culture medium using the soluble cellulose derivative, which acted coating the cellulosic nanofibers so that "coating islands" were formed, which strongly influenced the general properties of this biomaterial. It must be considered that the modifications exhibited by this new biocomposite is mainly a result of the viscosity increase of the culture medium exerted by the CMC addition, which directly interfered in the synthesis of the nanofibers and their final features, as evidenced previously by the various characterization techniques. In addition, the different DS of CMC plays a key role in modulating the fundamental properties of this biomaterial, since the $\mathrm{BC} / \mathrm{CMC}$ interaction pattern varies according to the number of carboxymethyl groups substituted by glucose residue in the CMC chains.

As is well known, the highly porous structure of BC does not constitute a barrier against the drug molecules diffusion, leading to the great majority of the release systems based on this biopolymer a burst effect of release. However, the lowering of the MTX release rates from BC/CMC0.9 in the end of test is an important suggestive that other DS of CMC, as well as its use in other amounts, should be considered in future studies, generating distinct biomaterials to be applied through the cutaneous route at different stages of evolution of a pathology, as psoriasis proposed in this study.

\section{Declaration of conflicts of interest}

The authors report no conflicts of interest associated with this publication.

\section{Acknowledgments}

The authors are thankful to National Counsel of Technological and Scientific Development MCTI/CNPQ/Universal 447601/2014-8. Moreover, we are grateful to the 'Macrobehavior- MesostructureNanotechnology' SGIker unit of the UPV/EHU.

\section{References}

Almeida, I. F., Pereira, T., Silva, N. H. C. S., Gomes, F. P., Silvestre, A. J. D., Freire, C. S. R., et al. (2014). Evaluation of the applied phosphate pectin in the formation of isolated films. Material candidate for new systems for modified drug release. European Journal of Pharmaceutics and Biopharmaceutics, 86, 332-336.

Andrade, F. K., Alexandre, N., Amorim, I., Gartner, F., Mauricio, A. C., Luis, A. L., et al. (2013). Studies on the biocompatibility of bacterial cellulose. Journal of Bioactive and Compatible Polymers, 28, 97-112.

Barud, H. S., Ribeiro, C. A., Crespi, M. S., Martines, M. A. U., Dexpert-Ghys, J., Marques, R. F. C., et al. (2007). Thermal characterization on bacterial cellulose-phosphate composite membranes. Journal of Thermal Analysis and Calorimetry, 87, 815-818.

Barud, H. S., Júnior, A. M. A., Santos, D. B., Assunção, R. M. N., Meireles, C. S., Cerqueira, D. A., et al. (2008). Thermal behavior of cellulose acetate produced from homogeneous acetylation of bacterial cellulose. Thermochimica Acta, 471, 61-69.

Boakye, C. H., Patel, K., Doddapaneni, R., Bagde, A., Marepally, S., \& Singh, M. (2017). Novel amphiphilic lipid augments the co-delivery of erlotinib and IL36 siRNA into the skin for psoriasis treatment. Journal of Controlled Release, 246, 120-132.

Bouklas, N., \& Huang, R. (2012). Swelling kinetics of polymer gels: Comparison of linear and nonlinear theories. Soft Matter, 8, 8194-8203.

Brannon-Peppas, L. (1990a). Preparation and characterization of crosslinked hydrophilic networks. In L. Brannon-Peppas, \& R. S. Harland (Eds.). Absorbent polymer technology (pp. 45-66). Amsterdam: Elsevier.

Brannon-Peppas, L. (1990b). The equilibrium swelling behavior of porous and non-porous hydrogels. In L. Brannon-Peppas, \& R. S. Harland (Eds.). Absorbent Polymer Technology (pp. 45-66). Amsterdam: Elsevier.

Caraschi, J. C., \& Campana, F. S. P. (1999). Influence of degree of substitution and substituent distribution on the carboxymethylcellulose equilibrium properties in aqueous solution. Polímeros, 9, 70-77.

Chai, M. N., \& Isa, M. I. N. (2013). Electrical characterization and ionic transport properties of carboxyl methylcellulose-oleic acid solid polymer electrolytes. International Journal of Polymer Analysis and Characterization, 18, 280-286.

Chawla, P. R., Bajaj, I. B., Shrikant, A., Survas, E., \& Singhal, R. S. (2008). Microbial cellulose: Fermentative production and applications. Food Technology and Biotechnology, 47, 107-124.

Chen, H.-H., Chen, L.-C., Huang, H.-C., \& Lin, S.-B. (2011). In situ modification of bacterial cellulose nanostructure by adding CMC during the growth of Gluconacetobacter xylinus. Cellulose, 18, 1573-1583.

Cheng, K.-C., Jefrrey, M. C., \& Demirci, A. (2009). Effect of different additives on bacterial cellulose production by Acetobacter xylinum and analysis of material property. Cellulose, 16, 1033-1045.

Cheng, K.-C., Catchmark, J. M., \& Demirci, A. (2011). Effects of CMC addition on bacterial cellulose production in a biofilm reactor and its paper sheets analysis. Biomacromolecules, 12, 730-736.

Credou, J., \& Berthelot, T. (2014). Cellulose: From biocompatible to bioactive material. Journal of Materials Chemistry B, 2, 4767-4788.

Donini, I. A. N., De Salvi, D. T. B., Fukumoto, F. K., Lustri, W. R., Barud, H. S., Marchetto, R., et al. (2010). Biosynthesis and recent advances in the production of bacterial cellulose. Eclética Química, 35, 165-178.

Fu, L., Zhang, Y., Li, C., Wu, Z., Zhuo, Q., Huang, X., et al. (2012). Skin tissue repair materials from bacterial cellulose by a multilayer fermentation method. Journal of Material Chemistry, 24, 12349-12357.

Gil, E. C., Colarte, A. I., Bataille, B., Brouillet, F., \& Caraballo, I. (2009). Estimation of the percolation thresholds in ternary lobenzarit disodium-dextran-HPMC hydrophilic matrices tablets: Effects of initial porosity. European Journal of Pharmeutical Sciences, $38,312-319$.

Goonoo, N., Jeetah, R., Luximon-Bhaw, A., \& Jhurry, D. (2015). Polydioxanone-based biomaterials for tissue engineering and drug gene delivery applications. European 
Journal of Pharmaceuticals and Biopharmaceuticals, 97, 371-391.

Hashem, M., Sharaf, S., El-Hady, M. M. E., \& Hebeish, A. (2013). Synthesis and characterization of novel carboxymethylcellulose hydrogels and carboxymethylcellulolsehydrogel-ZnO-nanocomposites. Carbohydrate Polymers, 95, 421-427.

Hirai, A., Tsuji, M., Yamamoto, H., \& Horii, F. (1998). In situ crystallization of bacterial cellulose III. Influences of different polymeric additives on the formation of microfibrils as revealed by transmission electron microscopy. Cellulose, 5, 201-213.

Huang, H.-C., Chen, L.-C., Lin, S.-B., Hsu, C.-P., \& Chen, H.-H. (2010). In situ modification of bacterial cellulose network structure by adding interfering substances during fermentation. Bioresource Technology, 101, 6084-6091.

Juncu, G., Stoica- Guzun, A., Stroescu, M., Isopencu, G., \& Jinga, S. I. (2016). Drug release kinetics from carboxymethylcellulose-bacterial cellulose composite films. International Journal of Pharmaceutics, 510, 485-492.

Kanamala, M., Wilson, W. R., Yang, M., Palmer, B. D., \& Wu, Z. (2016). Mechanisms and biomaterials in $\mathrm{pH}$-responsive tumour targeted drug delivery: A review. Biomaterials, 85, 152-167.

Kim, S. Y., Kim, J. N., Wee, Y. J., Park, D. H., \& Ryu, H. W. (2006). Production of bacterial cellulose by Gluconacetobacter sp. RKY5 isolated from persimmon vinegar. Applied Biochemistry and Biotechnology, 131, 705-715.

Li, X., Chen, S., Hu, W., Shi, S., Shen, W., Zhang, X., et al. (2009). In situ synthesis of CdS nanoparticles on bacterial cellulose nanofibers. Carbohydrate Polymers, 76, 509-512.

Li, Z., Wang, Y., Pei, Y., Xiong, W., Xu, W., Li, B., et al. (2017). Effect of substitution degree on carboxymethylcellulose interaction with lysozyme. Food Hydrodocolloids, $62,222-229$.

Lima, L. R., Santos, D. B., Barud, H. S., Henrique, M. A., Pasquini, D., Edison, P., et al. (2015). Nanocristais de celulose a partir de celulose bacteriana. Química Nova, 38, 1140-1147.

Liu, J., Zhan, X., Wan, J., Wang, Y., \& Wang, C. (2015). Review for carrageenan-based pharmaceutical biomaterials: Favourable physical features versus adverse biological effects. Carbohydrate Polymers, 121, 27-36.

Ma, T., Zhao, Q. Q., Ji, K. H., Zeng, B., \& Li, G. Q. (2014). Homogeneous and porous modified bacterial cellulose achieved by in situ modification with low amounts of carboxymethylcelulose. Cellulose, 21, 2637-2646.

Meneguin, A. B., Cury, B. S. F., \& Evangelista, R. C. (2014). Films from resistant starchpectin dispersions intended for colonic drug delivery. Carbohydrate Polymers, 99, $140-149$.

Meneguin, A. B., Cury, B. S. F., Santos, A. M., Franco, D. F., Barud, H. S., \& Filho, E. C. S. (2017). Resistant starch/pectin free-standing films reinforced with nanocellulose intended for colonic methotrexate release. Carbohydrate Polymers, 157, 1013-1023.

Moharram, M. A., \& Mahmoud, O. M. (2008). FTIR spectroscopic study of the effect of microwave heating on the transformation of cellulose I into cellulose II during mercerization. Journal of Applied Polymer Science, 107, 30-36.

Oh, S. Y., Yoo, D. I., Shin, Y., Kim, H. C., Kim, H. Y., Chung, Y. S., et al. (2005). Crystalline structure analysis of cellulose treated with sodium hydroxide and carbon dioxide by means of X-ray diffraction and FTIR spectroscopy. Carbohydrate Research, 340, 2376-2391.

Ribeiro, C., Sencadas, V., Correia, D. M., \& Lanceros-Méndez, S. (2015). Piezoelectric polymers as biomaterials for tissue engineering applications. Colloids and Surfaces B:
Biointerfaces, 136, 46-55.

Ritger, P. L., \& Peppas, N. A. (1987). A simple equation for description of solute release II. Fickian and anomalous release from swellable devices. Journal of Controlled Release, 5, 37-42.

Schenck, T.-L., Hopfner, U., Chávez, M.-N., Machens, H.-G., Somlai-Schweiger, I., Giunta, R.-E., et al. (2014). Photosynthetic biomaterials: A pathway towards autotrophic tissue engineering. Acta Biomaterialia, 15, 39-47.

Segal, L., Creely, J. J., Martin, A. E., \& Conrad, C. M. (1959). An empirical method for estimating the degree of crystallinity of native cellulose using the X-ray diffractometer. Textile Research Journal, 29, 786-794.

Shoda, M., \& Sugano, Y. (2005). Recent advances in bacterial cellulose production. Biotechnology and Bioengineering, 10, 1-8.

Siepmann, J., \& Peppas, N. A. (2011). Higuchi equation: Derivation, applications, use and misuse. International Journal of Pharmaceutics, 418, 6-12.

Silva, N. H. C. S., Rodrigues, A. F., Almeida, I. F., Costa, P. C., Rosado, C., Neto, C. P., et al. (2014). Bacterial cellulose membranes as transdermal delivery systems for diclofenac: In vitro dissolution and permeation studies. Carbohydrate Polymers, 106, 264-269.

Sinha, V. R., \& Kumria, R. (2003). Microbially triggered drug delivery to the colon. European Journal of Pharmaceutical Sciences, 18, 3-18.

Souto-Maior, J. F. A., Reis, A. V., Pedreiro, L. N., \& Cavalcanti, O. A. (2017). Avaliação da pectina fosfatada aplicada na formação de filmes isolados. Material candidato a novos sistemas para liberação modificada de fármacos. Brazilian Journal of Pharmaceutical Sciences, 44, 203-213.

Stumpf, T. R., Yang, X., Zhang, J., \& Cao, X. (2016). In situ and ex situ modifications of bacterial cellulose for applications in tissue engineering. Materials Science and Engineering: C. http://dx.doi.org/10.1016/j.msec.2016.11.121.

The United States Pharmacopeia (2006). United States pharmacopoeial convention (29th ed.). Rockville: The United States Pharmacopeia.

Trovatti, E., Freire, C. S. R., Pinto, P. C., Almeida, I. F., Costa, P., Silvestre, A. J. D., et al. (2012). Bacterial cellulose membranes applied in topical and transdermal delivery of lidocaine hydrochloride and ibuprofen: In vitro diffusion studies. International Journal of Pharm Aceutics, 435, 83-87.

Voigt, R. (2000). Pharmazeutische technologie: für Studium und Beruf (9th ed.). Deutscher Apotheker: Stuttgart.

Wang, X., \& Yan, H. (2017). Methotrexate-loaded porous polymeric adsorbents as oral sustained release formulations. Materials Science and Engineering, 78, 598-602.

Wang, W.-Y., Zhao, X.-F., Ju, X.-H., Wang, Y., Wang, L., Lia, S.-P., et al. (2016). Novel morphology change of Au-methotrexate conjugates: From nanochains to discrete nanoparticles. International Journal of Pharmaceutics, 515, 221-232.

Wei, W., Jing, L., Shuqin, C., Hong, T., Jianshu, L., Fujian, X., et al. (2011). Drug release behaviors of a $\mathrm{pH}$ sensitive semi-interpenetrating polymer network hydrogel composed of poly(vinyl alcohol) and star poly[2-(dimethylamino)ethyl methacrylate]. International Journal of Pharmaceutics, 416, 104-109.

Yamamoto, H., Horii, F., \& Hirai, A. (1996). In situ crystallization of bacterial cellulose II. Influences of polymeric additives with different molecular weights on the formation of celluloses I $\alpha$ and I $\beta$ at the early stage of incubation. Cellulose, 3, 229-242. 